\title{
LATERALIDADE E FRACASSO ESCOLAR
}

\author{
VIANNA, José Antônio ${ }^{1}$
}

\section{RESUMO}

As restrições às vivências motoras decorrentes das transformações nos modos de vida da sociedade atual parecem comprometer o desenvolvimento motor de crianças em idade escolar. Este estudo procurou comparar a lateralidade de alunos com desempenho escolar regular (DR) com a lateralidade de alunos em situação de fracasso escolar (FE). Foram avaliados 89 sujeitos entre 8 anos e 13 anos de idade $(M=10,4)$. Ambos os grupos foram submetidos ao Harris Test of Lateral Dominance. Os resultados indicaram que $53,2 \%$ dos sujeitos do grupo FE e $57,2 \%$ do grupo DR apresentaram lateralidade indefinida. O teste qui-quadrado $(p=0,05 / g l=2)$ não observou diferença significativa entre os grupos investigados.

PaLAVRas-Chave: Lateralidade - Fracasso escolar - Desenvolvimento motor - Dificuldades de aprendizagem.

\section{ABSTRACT}

Restrictions on motor experiences resulting from changes in the lifestyles of today's society seem to impair the motor development of school-age children. The aim of this study was to compare the laterality of students with regular school to the laterality of students in a situation of school failure. Were evaluated 89 subjects between 8 years and 13 years of age (M $=10.4)-$ male and female students in a school in Rio de Janeiro - which were submitted to the Harris Test of Lateral Dominance. $53,2 \%$ of subjects in the FE group and $57,2 \%$ of the DR group had laterality undefined between the groups investigated.

KEYWORDS: Laterality - School failure - Motor Development - Learning difficulties.

\footnotetext{
${ }^{1}$ Professor Adjunto do Instituto de Educação Física e Desportos (DESCO/UERJ) e Professor do Programa de Pósgraduação em Educação Básica (PPGEB/CAp-UERJ). Email: javianna2@hotmail.com
} 


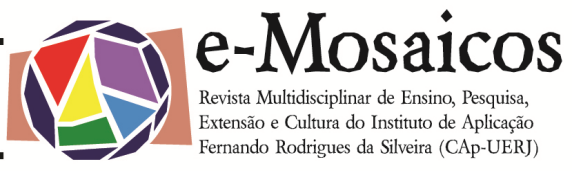

\section{INTRODUÇÃO}

$\mathrm{O}$ aluno em situação de fracasso escolar (FE) pode ser identificado como o sujeito com idade cronológica incompatível com a idade escolar e com dois ou mais anos de reprovação escolar. O FE tem sido investigado sob a perspectiva psicológica, como um problema técnico, como questão institucional, como uma questão política, entre outras (ANGELUCCI et al, 2004).

As dificuldades na aquisição da leitura e da escrita chegam a atingir $25 \%$ dos alunos que apresentam diferenças importantes entre o desempenho esperado e o desempenho que apresentam durante o processo. Existem evidências de que crianças que não tiveram experiências ricas de movimento corporal podem ter 0 seu desenvolvimento motor comprometido e apresentar dificuldades de aprendizagem devido a distúrbios ou transtornos de aprendizagem (FONSECA, 1998; GALLAHUE; OZMUN, 2005, LE BOUCH, 2008, ROSA NETO, 2002; LUCENA et al, 2010).

Por outro lado, nota-se, também, a presença de alunos matriculados regularmente nos vários anos (séries) de escolaridade, que são analfabetos ou analfabetos funcionais, mesmo sem serem portadores de qualquer incapacidade (DINIZ; MEDEIROS; SQUINCA, 2007) ou distúrbios de aprendizagem. No entanto, as pesquisas têm priorizado sujeitos com distúrbios ou patologias que comprometem o aprendizado em detrimento dos sujeitos que, sem diagnóstico de dificuldades de aprendizagem, encontram-se em situação de fracasso escolar.

A relação positiva entre a atividade física e o desempenho escolar foi observada em investigações que confirmaram a ligação entre a atividade física, a cognição e a performance escolar (DONELLY; LAMBOURNE, 2011), a melhoria na saúde mental de alunos em anos iniciais de escolaridade (AHN; FEDEWA, 2011), a importância do desenvolvimento de habilidades motoras na Educação Infantil para o desenvolvimento de habilidades matemáticas e de habilidades verbais (PAGANI; MESSIER, 2012) e a associação entre tempo gasto em aulas de Educação Física e a melhoria no desempenho escolar de alunas em matemática e leitura (CARLSON, 2008).

Para haver o desenvolvimento de habilidades necessárias à aprendizagem escolar, é preciso que sejam dadas à criança oportunidades de movimentar o próprio corpo. Pelo movimento o sujeito desenvolve capacidades fundamentais para a aprendizagem escolar, entre as quais a lateralidade (PACHER et al., 2003). Da noção dos dois lados do corpo, esquerdo e direito, será desenvolvida a orientação espacial frente a objetos, imagens e símbolos, sem a qual a aprendizagem escolar pode se tornar mais difícil (THOMÉ et al., 2010).

Assim, uma área importante de investigação é identificar a lateralidade de alunos do primeiro segmento do ensino fundamental matriculados em uma escola pública e comparar a lateralidade de alunos com características de fracasso escolar com a lateralidade de escolares com desempenho escolar dentro da normalidade.

\section{O CONTEXTO TEÓRICO}

A importância da Educação Física (EF), no meio escolar, está em proporcionar o ambiente apropriado para a criança experimentar, vivenciar, conhecer as possibilidades e limitações do próprio corpo. Sob esta perspectiva, a EF escolar é assistente e motivadora do desenvolvimento integral do ser humano (SCHIRMER; FONTOURA; NUNES, 2004).

Admite-se que a educação do movimento interfere positivamente no pensamento, no conhecimento, na ação - nos domínios cognitivos - e na vida do ser humano. Para ter uma vida ativa, saudável e produtiva, com o desenvolvimento integral 
e-Mosaicos - Revista Multidisciplinar de Ensino, Pesquisa, Extensão e Cultura do Instituto de Aplicação Fernando Rodrigues da Silveira (CAp-UERJ)

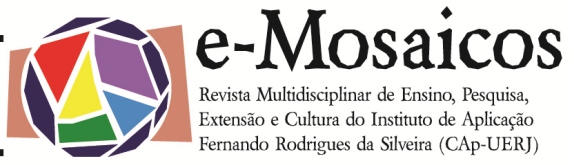

apropriado, é necessário que o indivíduo esteja fisicamente educado (MONTEIRO, 2007).

A Educação Física escolar pode contribuir para o desenvolvimento integral dos sujeitos quando abordam os gestos esportivos, os movimentos da cultura corporal humana (SCHIRMER; FONTOURA; NUNES, 2004) e outros aspectos motores, levando em conta que o mesmo integra o ato físico relacionado ao trabalho mental, formando bases essenciais para o aprendizado escolar (ROSA NETO et al., 2007).

O desenvolvimento motor é de extrema importância para a formação dos indivíduos, para o desenvolvimento funcional e para a possível expansão de sua afetividade (DARIDO; RANGEL, 2005). O desenvolvimento motor é essencial para o desenvolvimento integral harmonioso e para evitar o aparecimento de problemas de aprendizagem. Consequentemente, a motricidade pode cooperar com o processo de aprendizagem escolar através das aulas de Educação Física (MONTEIRO, 2007), ao interceder por uma postura educativa que deve acontecer por meio de movimentos corporais realizados pela criança, beneficiando a formação da imagem corporal - principal peça da personalidade (DARIDO; RANGEL, 2005).

Segundo Fonseca (1998), o desenvolvimento do cérebro se dá graças à motricidade humana. $\mathrm{O}$ autor argumenta que é possível a existência da motricidade sem desenvolvimento cognitivo, mas desenvolvimento cognitivo sem motricidade humana não é. Assim, para um desenvolvimento intelectual ajustado, deve haver um desenvolvimento adequado da motricidade (FONSECA, 1998). Esta interface sugere que possíveis deficiências no desenvolvimento motor contribuem para um desenvolvimento inferior da linguagem e da cognição.

Desta forma, as experiências motoras são alicerce para a educação no ensino fundamental. É imprescindível que a crian- ça tenha vivenciado a cor, espessura, longitude, latitude, trajetória, ângulo, forma, orientação e projeção espacial, antes que se tenha iniciado o processo de alfabetização (BRÊTAS, 2005). Para prevenir que crianças manifestem comprometimento de habilidades escolares, deve-se propiciar o maior número possível de vivências motoras e psicossociais (ROSA NETO et al., 2007).

Algumas das dificuldades de aprendizagem da leitura e escrita apresentadas por crianças de 6 a 7 anos, quando as mesmas começam a ser alfabetizadas, são decorrentes de uma experiência motora pobre. Segundo Kalakian e Goldman (citado por TANI et al., 1988), a lateralidade, a imagem corporal, o equilíbrio, a locomoção e a percepção têm um papel importante no desenvolvimento cognitivo. Um dos principais fatores responsáveis pelo desenvolvimento das capacidades de aprendizagem escolar é a lateralidade. A lateralidade da criança começa a se definir durante o crescimento, e pode, também, ser influenciada por fatores sociais (PACHER et al., 2003).

Alguns erros são mais frequentes, como os de inversão e espelhamento, e ocorrem tanto na leitura como na escrita: conflito entre letras simétricas $(p / q ; n / u$; $\mathrm{d} / \mathrm{b}$ e $\mathrm{g} / \mathrm{q}$ ); troca da ordem das letras dentro de uma sílaba (pal / pla) e troca de ordem das sílabas numa palavra (aeroplano / areoplano) (ZORZI, 2000). A criança que não consegue diferenciar esquerda e direita pode não ser capaz de respeitar a direção gráfica da leitura começando pela esquerda (LUCENA et al, 2010).

Podem ser encontrados na literatura três tipos de lateralidade: lateralidade definida, lateralidade cruzada e lateralidade indefinida (VIEIRA; CAVALLI, 1997). Quando mão, olho e pé respeitam o mesmo lado do corpo, a dominância lateral é definida, e é caracterizada pela dominância de um hemisfério cerebral. Já a dominância lateral cruzada encontra-se um dos 
e-Mosaicos - Revista Multidisciplinar de Ensino, Pesquisa, Extensão e Cultura do Instituto de Aplicação Fernando Rodrigues da Silveira (CAp-UERJ)

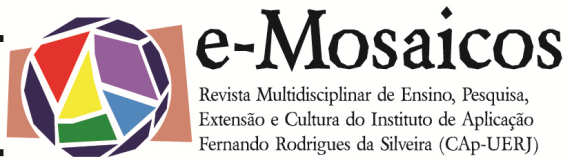

órgãos discordando em relação ao lado dominado pelos outros. Na dominância lateral não definida, a dominância é desarmônica num mesmo órgão (BRÊTAS, 2005).

Os distúrbios de lateralidade podem ser associados aos problemas de aprendizagem escolar. Harris, em 1957, ligou a desordem na dominância cerebral com distúrbios para leitura. Em 1971, Barreto, descobriu haver associação entre lateralidade cruzada e dificuldades de aprendizagem. Rebello, em 1967, revelou haver constância de crianças com lateralidade cruzada ou mal definida simultaneamente com disfunção cerebral mínima em clínica neuropediátrica (PACHER et al., 2003).

Existem evidências de que a lateralidade participa no processo de aprendizagem, mas ela só se estabelece terminantemente nos primeiros anos escolares (LUCENA et al, 2010). É neste período que o esquema corporal e a organização espacial se constituem, dando à criança a capacidade de compreender as noções externas (direita, esquerda) pertencentes ao próprio corpo (MACEDO; ANDREUCCI; MONTELLI, 2004). Dessas noções advêm as relações de orientação espacial frente aos objetos e imagens. Portanto, qualquer criança que demonstre problemas para dominar estas noções pode revelar dificuldades no traçado e na combinação de letras e números (LUCENA et al., 2010).

Agregados às dificuldades de leitura e escrita, normalmente se encontram outros distúrbios, como: distúrbios da noção de espaço; distúrbios do esquema corporal e do sentido de direção; distúrbios da noção de tempo e distúrbios da percepção do ritmo (ZORZI, 2000). Esses distúrbios são vistos como fatores que resultam em problemas apresentados na leitura e na escrita.

Este trabalho teve como objetivos verificar a dominância lateral de alunos do primeiro segmento do ensino fundamental de uma escola pública no município do Rio de Janeiro e comparar a dominância lateral de alunos em situação de Fracasso Escolar (FE) e de alunos com desempenho escolar dentro da normalidade, chamados aqui de sujeitos com Desempenho Regular (DR), tendo como base os resultados da aplicação do Harris Test of Lateral Dominance adaptado de Lucena et al. (2010).

\section{Metodologia}

\section{Sujeitos investigados}

Foram identificados em uma escola pública situada no interior de um bairro popular no município do Rio de Janeiro, 47 alunos com idade cronológica incompatível com a idade escolar e com histórico de dois ou mais anos de defasagem escolar enquadrados neste estudo no grupo com características de fracasso escolar (FE), sendo 20 do sexo feminino e 27 do sexo masculino, sem qualquer diagnóstico de patologia que pudesse comprometer a aprendizagem escolar. Estes indivíduos foram submetidos ao Harris Test of Lateral Dominance adaptado de Lucena et al. (2010) para a avaliação da dominância lateral. A lateralidade do grupo FE foi comparada com a lateralidade de 42 indivíduos que se submeteram voluntariamente para participar da pesquisa - Foram selecionados para este grupo sujeitos classificados como alunos com desempenho regular (DR) por terem obtido conceitos $R$, $B$ ou MB na prova aplicada pela prefeitura a alunos da rede municipal de ensino -17 do sexo feminino e 25 do sexo masculino. Os estudantes investigados estavam situados entre 8 anos e 13 anos de idade ( $M=$ $10,4)$.

\section{Procedimentos}

Foram observadas as dominâncias de mãos, olhos e membros inferiores. Para a avaliação da dominância lateral de mãos foram utilizados os seguintes objetos: bola de tênis, relógio de corda, martelo e 
prego, escova de dentes, pente, porta em miniatura, folha de papel A4, lápis, tesoura e caneta. Para a avaliação da dominância lateral de olhos foram utilizados: cartão de cartolina preta com um orifício de $0,5 \mathrm{~cm}$, um tubo largo com $10 \mathrm{~cm}$ de diâmetro e um caleidoscópio. Para a verificação da dominância lateral de membros inferiores foi utilizada uma bola.

A coleta de dados só teve início após a aprovação e autorização da coordenadora pedagógica e da diretora da escola. Em seguida os Termos de Consentimento Livre e Esclarecido foram entregues às educadoras, com o intento de que fosse repassado aos pais das crianças. As crianças cujos responsáveis assinaram TCLE participaram do estudo, que necessitou de três dias para avaliar todos os participantes.

A avaliação de cada criança aconteceu em uma sala especialmente preparada para a aplicação do teste a fim de que não ocorressem interferências externas. Em seguida, como orientação, foi explicada para a criança o que deveria ser feito. Em caso de dúvida explicava-se novamente, até que não houvesse mais dúvidas.

Durante todo o teste as crianças permaneceram em pé, elas passavam de estágio para estágio, se colocando em frente e de frente para os objetos.

Para verificar a lateralidade manual, as crianças passaram pelos seguintes estágios: (10 estágios) - lançar uma bola, dar corda num relógio, martelar um prego, escovar os dentes, pentear o cabelo, virar maçaneta, pintar, recortar, cortar um pão e escrever.

Para a lateralidade ocular, primeiramente o examinador demonstrava para a criança como o procedimento deveria ser feito, segurando o cartão de cartolina com as duas mãos e com braços estendidos, aproximando-os lentamente ao rosto em direção a um dos olhos. Após, foi pe- dido à criança para realizar a mesma tarefa. A mesma técnica foi utilizada com o tubo e, em seguida, com o caleidoscópio.

Para observar a lateralidade de membros inferiores, as crianças passaram por dois estágios: colocar um pé sobre a bola e chutá-la.

Após cada estágio era anotado o lado utilizado pela criança para realizar a tarefa, direito ou esquerdo. Cada parte era testada isoladamente (mão, olho e pé). Sempre que todas as tarefas de uma parte eram realizadas com o mesmo lado anotava-se com letra maiúscula, (D) direita ou (E) esquerda. Se a criança realizasse pelo menos uma tarefa com o lado diferente das demais, anotava-se com letra minúscula (d ou e).

Ao final dos testes obteve-se uma fórmula de três letras, cada uma respondendo à dominância manual, ocular e dos membros inferiores, respectivamente. Foram consideradas com lateralidade definida direita-esquerda as crianças que obtiveram uma fórmula com três letras maiúsculas iguais (DDD ou EEE), com lateralidade cruzada as crianças que obtiveram duas letras maiúsculas iguais e uma diferente (EDD. EED, EDE, DEE, DDE, DED) e com lateralidade indefinida quando houve presença de uma letra minúscula ( $d E E, D d D$, EEd e etc.).

\section{Análise dos dados}

Para verificar a existência de diferença significativa na lateralidade de alunos com fracasso escolar (FE) e alunos com desempenho escolar dentro da normalidade (DR) foi aplicado o teste Quiquadrado ao nível de significância de 0,05 (LEVIN, 1987).

O estudo foi aprovado pelo Comitê de Ética em Pesquisa, protocolo no CAAE 0022.0.308.000-11 - todos os responsáveis pelos participantes assinaram um Termo de Consentimento Livre e Esclarecido antes de sua inclusão na amostra. 
e-Mosaicos - Revista Multidisciplinar de Ensino, Pesquisa, Extensão e Cultura do Instituto de Aplicação Fernando Rodrigues da Silveira (CAp-UERJ)

Embora com média de idade superior (10,4 anos) ao que preconiza a literatura para a definição da lateralidade, menos da metade de todos os participantes investigados apresentaram dominância lateral definida $(31,9 \%)$ ou dominância cruzada $(15,7 \%)$. A maioria dos sujeitos investigados $(52,4 \%)$ apresentou a lateralidade indefinida.

No grupo de 42 indivíduos classificados como alunos com desempenho re- gular (DR), 4 sujeitos apresentaram dominância lateral cruzada, 14 dominância lateral direita e 24 dominância indefinida.

A maior parte dos sujeitos do grupo FE apresentaram lateralidade indefinida $(53,2 \%)$ e $57,2 \%$ do grupo DR tiveram a mesma particularidade. Foram identificados $14,9 \%$ de alunos FE com lateralidade cruzada, enquanto $9,5 \%$ dos indivíduos com DR mostraram esta característica. (Tabela 1).

Tabela 1 - Frequência absoluta e relativa da dominância lateral dos grupos FE e DR.

\begin{tabular}{|c|c|c|c|c|}
\hline \multirow[t]{2}{*}{ Dominância } & \multicolumn{2}{|c|}{ FE } & \multicolumn{2}{|c|}{ DR } \\
\hline & Freq. & $\%$ & Freq. & $\%$ \\
\hline $\begin{array}{l}\text { Destro completo } \\
\text { Sinistro completo }\end{array}$ & 15 & 31,9 & 14 & 33,3 \\
\hline Lateralidade cruzada & 7 & 14,9 & 4 & 9,5 \\
\hline Lateralidade indefinida & 25 & 53,2 & 24 & 57,2 \\
\hline Total & 47 & 100 & 42 & 100 \\
\hline
\end{tabular}

Para verificar se existe diferença significativa entre os grupos investigados foi utilizado o teste qui-quadrado ao nível de significância de 0,05 e gl = 2. A aplicação do teste não encontrou discrepância entre os grupos $\left(\mathrm{X}^{2} \mathrm{O}=0,583<\mathrm{X}^{2} \mathrm{e}=\right.$ $5,99)$.

\section{DISCUSSÃo}

Ao analisar a dominância lateral de alunos no primeiro segmento do ensino fundamental, em uma escola pública situada em um bairro popular no município do Rio de Janeiro, pode-se constatar que a maioria dos indivíduos investigados não estava com a lateralidade definida, apesar da idade média do grupo estar em 10,4 anos. Já que o cérebro se desenvolve no sentido de direcionalidade e lateralidade a partir dos 6 anos de idade - crianças acima de 6 anos, deveriam apresentar na sua maioria lateralidade definida, tendo desenvolvido o sentido de direcionalidade e lateralidade pelo cérebro (THOMÉ et al, 2010) - este dado sugere que os sujeitos investigados podem ter dificuldades em aprender (CONOLLY, 1983).

Ao considerar a importância da definição da dominância lateral na aquisição de habilidades necessárias para a leitura e a escrita (ZORZI, 2000; LUCENA et al., 2010), admite-se que parte significativa dos investigados pode não ser capaz de respeitar a direção gráfica da leitura e ter dificuldades no traçado e na combinação de letras e números (LUCENA et al., 2010).

Os resultados indicaram que a indefinição na dominância lateral direitaesquerda é um fator predominante nos grupos investigados. Mais da metade dos indivíduos em ambos os grupos apresentaram a lateralidade indefinida. Ao que tudo indica, a lateralidade indefinida não é uma particularidade dos sujeitos com fracasso escolar. Os alunos da escola pública com desempenho escolar normal que não tiveram vivências motoras ricas e diversificadas podem ter a lateralidade indefinida.

Considera-se que a noção dos dois 
lados do corpo, esquerdo e direito, tendo como referência a linha média do corpo é representada pela lateralidade. Dessa noção são desenvolvidas as ligações entre orientação frente a objetos, imagens e símbolos. Assim, a lateralidade vai influir diretamente na aprendizagem escolar (THOMÉ et al., 2010). O que não significa que todas as crianças que têm dificuldades de aprendizagem também tenham déficit na lateralidade, mas como o que pode ser verificado em ambos os grupos estudados, mais da metade dos sujeitos não apresentaram a lateralidade definida.

Chama a atenção, nos dados coletados, a ausência de sinistros completos, o que leva ao questionamento se parte dos indivíduos com lateralidade indefinida seja devido à pressão social que induz as crianças à utilização dos membros direitos em tarefas cotidianas.

$\mathrm{O}$ teste de qui-quadrado confirmou a inexistência de diferença significativa na lateralidade dos alunos participantes nos grupos FE e DR. Acredita-se que os alunos que apresentaram melhor desempenho escolar lançam mão de outras habilidades e competências para suprir as limitações decorrentes da lateralidade indefinida.

As informações coletadas por Rasberry et al (2011) que verificaram 251 associações entre as atividades de Educação Física e o desempenho escolar, sendo mais de $50 \%$ positivas e apenas $1,5 \%$ negativas, os dados que ligam a atividade física e o bom desempenho acadêmico (DONELLY; LAMBOURNE, 2011) e a meIhoria da saúde mental (AHN; FEDEWA, 2011), sustentam as informações coletadas em crianças com ou sem lateralidade cruzada e crianças com dificuldades de aprendizagem submetidas a um plano de exercícios físicos que indicaram melhora na definição da lateralidade dos alunos. Embora Sanches et al (2004) não tenham encontrado relação da lateralidade cruzada ou indefinida com o rendimento escolar, e também não tenha observado desarmonia entre a lateralidade e o desempenho escolar (SANCHES et al., 2004). Por outro lado, Gardiola, Ferreira e Rotta (1998), demonstraram ligação entre a lateralidade mal estabelecida e problemas de linguagem ou dificuldades escolares, o que reforça a tese da importância da prática orientada de Educação Física na escola (CARLSON, 2010).

Diferente dos distúrbios patológicos que comprometem a aprendizagem, muitas crianças em idade escolar chegam à escola com deficiências no que diz respeito à aquisição de habilidades motoras fundamentais (OLIVEIRA, 2002), devido a vivências em ambientes físico e sociais que não contribuíram para o enriquecimento do repertório motor destes sujeitos. As experiências de vida parecem determinar a formação de sujeitos com mais ou menos possibilidades de transformar a sua própria vida e contribuir para a melhoria do meio social humano. Desta forma, as condições das crianças em casa e na escola, podem oferecer condições suficientes ou insuficientes para a formação adequada dos jovens. Uma formação não adequada pode significar em dificuldades de aprendizagem escolar mesmo em alunos sem diagnóstico de patologias, ou seja, em escolares normais que não tiveram estímulos suficientes para o seu desenvolvimento motor pleno.

Se por um lado os distúrbios de aprendizagem estão relacionados a patologias decorrentes de disfunções biológicas no sistema nervoso central, por outro lado, as dificuldades de aprendizagem ocorrem por problemas ou limitações nas experiências de vida e / ou no processo de ensino aprendizagem e podem ser mais facilmente removidas (TULESKI; EIDT, 2007).

\section{CONCLUSÕES E RECOMENDAÇÕES}

O desempenho escolar dos participantes nesta investigação sustenta outras pesquisas que alertam para a necessidade 
de esforços interdisciplinares e multiprofissionais para a melhoria da qualidade da educação pública e gratuita. Os achados deste estudo indicam que a lateralidade não foi o único fator para a presença de dificuldades de aprendizagem ou fracasso escolar, embora a literatura informe que a lateralidade mal definida influencie no processo de aprendizagem escolar.

Por razões sociais ou econômicas, o crescimento dos centros urbanos tem limitado as oportunidades de experimentação e expressão corporal, com prejuízos ao desenvolvimento integral dos futuros cidadãos - o mesmo parece estar ocorrendo nos bairros populares. Dessa forma, ao sistematizar as atividades perceptivomotoras a Educação Física escolar pode ajudar os alunos a consolidarem suas habilidades e competências - equilibrando 0 desenvolvimento filogênico e ontogênico para ultrapassar as limitações e dificuldades ocasionadas por ambientes sociais e culturais inadequados e precaver inadaptações, com o propósito de oferecer condições ótimas para um bom desempenho escolar.

Acima da idade sugerida para a consolidação da dominância lateral direitaesquerda, a maioria dos sujeitos investigados não apresentou definição na lateralidade. A análise estatística não observou diferença entre os grupos investigados. Assim, acredita-se que a diferença no rendimento escolar pode ser devido a fatores sociais, como apoio familiar, acesso a bens sociais e culturais, afazeres domésticos, trabalho infantil, formação escolar deficiente, entre outros.

Um programa de Educação Física bem estruturado pode garantir um desenvolvimento harmonioso dos alunos e minimizar os efeitos derivados da socialização inadequada. Sendo assim, há necessidade de se introduzir no cotidiano escolar atividades de estimulação motora específicas à lateralidade funcional de crianças, com o objetivo de prevenir e intervir em prováveis distorções decorrentes do processo de desenvolvimento e aprendizagem desde os primeiros anos de vida.

Investigar alunos de contextos sociais e culturais diferentes e com desempenho escolar distintos pode contribuir para ampliar a compreensão do fenômeno em pauta.

\section{REFERÊNCIAS BIBLIOGRÁFICAS}

AHN, S.; FEDEWA, A. L. A Meta-analisys of the relationship between children's physical activity and mental health. Journal of Pediatric Psychology. vol. 36, n04, 2011.

ALAVARSE, O. M. A organização do ensino fundamental em ciclos: algumas questões. Revista Brasileira de Educação. vol.14, 2009.

ANGELUCCI, C. B. et al. O estado da arte da pesquisa sobre 0 fracasso escolar (1991-2002): um estudo introdutório. Educação e Pesquisa. vol.30, 2004.

BRÊTAS, José Roberto. Avaliação de funções psicomotoras em crianças entre 6 e 10 anos de idade. Acta Paulista de Enfermagem vol. 18, n04, São Paulo, out-dez, 2005. http://dx.doi.org/10.1590/S010321002005000400009

CARLSON, S. Physical education and academic achievement in elementar school: data from the early childhood longitudinal study. American Journal of Public Health. vol.98, n04, 2008.

DALBEN, A. I. L. F. Os ciclos de formação como alternativa para a inclusão escolar. Revista Brasileira de Educação. vol.14, 2009.

DARIDO, Suraya Cristina; RANGEL, Irene Conceição. Educação Física na Escola: Implicações para a Prática Pedagógica. Rio de Janeiro: Guanabara Koogan, 2005.

DINIZ, D.; MEDEIROS, M.; SQUINCA, F. 
Reflexões sobre a versão em Português da Classificação Internacional de Funcionalidade, Incapacidade e Saúde. Cadernos de Saúde Pública. vol.23, n¹0, Rio de Janeiro, $\quad 2007$. http://dx.doi.org/10.1590/S0102311X2007001000025.

DONELLY, J. E.; LAMBOURNE, K. Classroom-based physical activity, cognition, and academic achievement. Preventive Medicine. vol. 52, 2011.

FIGUEIREDO, I. M. Z. Os projetos financiados pelo banco mundial para o ensino fundamental no Brasil. Educação e Sociedade. vol.30, 2009.

FONSECA, Vitor. Psicomotricidade: Filogênese, Ontogênese e Retrogênese. 2. ed. Porto Alegre: Artmed, 1988.

GALLAHUE, David L.; OZMUN, John C. Compreendendo o Desenvolvimento Motor: Bebês, Crianças e Adultos. 3. ed. São Paulo: Phorte, 2005.

GUARDIOLA, Ana; FERREIRA, Lúcia Teresinha; ROTTA, Newra Tellechea. Associação entre Desempenho das Funções Corticais e Alfabetização em uma Amostra de Escolares de Primeira Série de Porto Alegre. Arquivos de Neuro-psiquiatria. vol. 56, n02, jun. pp. 281-288, 1998.

LUCENA, Neide Maria et al. Lateralidade Manual, Ocular e dos Membros Inferiores e sua Relação com déficit de organização espacial em escolares. Estudos de Psicologia, Campinas, vol. 27, n01. jan/mar. pp. 3-11, 2010. http://dx.doi.org/10.1590/S0103166X2010000100001

LEVIN, Jack. Estatística aplicada a ciências humanas. 2ed. São Paulo: Harbra, 1987.

MACEDO, Célia Sperandéo; ANDREUCCI, Lívia Christina; MONTELLI, Teresina de Cresci. Alterações Cognitivas em Escolares de Classe Sócio-econômica Desfavorecida:
Resultados de Intervenção Psicopedagógica. Arquivos de Neuro-psiquiatria, São Paulo, Botucatu, vol. 62, n03b, pp. 852857, 2004. http://dx.doi.org/10.1590/S0004282X2004000500021

MONTEIRO, Vanessa. A psicomotricidade nas aulas de Educação Física escolar: Uma ferramenta de auxílio na aprendizagem. EF Desportes: Revista digital, Buenos Aires, ano. 12, n.114, 2007. Recuperado em: outubro 18, 2010, disponível em <http://www.efdeportes.com/efd114/apsicomotricidade-nas-aulas-de-educacaofisica-escolar.htm>.

OLIVEIRA, J. Padrões motores fundamentais: implicações e aplicações na educação infantil. Interação. Ano II, vol.6, dez, 2002.

PACHER, Luciana et al. Lateralidade e Educação Física. Revista Inspirar. Blumenau, vol.1, n03, pp.1, ago - dez, 2003.

PAGANI, L. S.; MESSIER, S. Links between motor skills and indicators of school readiness at kindergarten entry in urban disadvantaged children. Journal of Educational and Developmental Psychology. vol.2, no1, 2012. DOI: $10.5539 /$ jedp.v2n1p95

ROSA NETO, Francisco et al. Desenvolvimento Motor de Crianças com Indicadores de Dificuldades na Aprendizagem Escolar. Revista Brasileira de Ciência e Movimento, Florianópolis,vol. 15, n01, pp. 45-51, 2007.

ROSA NETO, F. et al. O esquema corporal de crianças com dificuldade de aprendizagem. Revista Semestral da Associação Brasileira de Psicologia Escolar e Educacional, SP. vol.15, n01, jan/jun 2011. Disponível em: http://dx.doi.org/10.1590/S14135572011000100002.

RASBERRY, C. N. et al. The association between school-based physical activity, including physical education, and academ- 
e-Mosaicos - Revista Multidisciplinar de Ensino, Pesquisa, Extensão e Cultura do Instituto de Aplicação Fernando Rodrigues da Silveira (CAp-UERJ)

ic performance: A systematic review of the literature. Preventive Medicine, vol. 52, 2011.

SANCHES, Sabrina de Oliveira et al. Perfil Psicomotor Associado a Aprendizagem Escolar. EF Desportes: Revista Digital, Buenos Aires, ano.10, n.79, 2004. Recuperado em: outubro 16, 2010, disponível em $<$ http://www.efdeportes.com/efd79/psico m.htm>.

SCHIRMER, Carolina; FONTOURA, Denise; NUNES, Magda. Distúrbios da Aquisição da Linguagem e da Aprendizagem. Jornal de Pediatria, Porto Alegre, vol.80, n02, supl., pp. 95-103, 2004. ISSN 0021-7557. http://dx.doi.org/10.1590/S002175572004000300012.

TANI, G.; MANOEL, E. J.; KOKUBUM, E.; PROENÇA, J. E. Educação física escolar. fundamentos de uma abordagem desenvolvimentista. São Paulo: EPU: EDUSP, 1998.

THOMÉ, Kélin et al. Avaliação do equilíbrio e lateralidade em crianças pré-escolares. Revista Inspirar, Curitiba, vol. 2, n04, pp. 24-33, jan-fev, 2010.

TULESKI, Silvana Calvo; EIDT, Nadia Maria. Repensando os distúrbios de aprendizagem a partir da psicologia históricocultural. Psicologia em Estudo, Maringá, vol.12, n03, pp. 531-540, set/dez, 2007.

VIEIRA, Lemanar Fiorese; CAVALLI, Marlene Gesualdo. Estudo da Lateralidade em Pré-escolares de 4 a 6 anos da Escola Benedito de Souza da Rede Municipal de Ensino e Maringá - PR. Revista da Educação Física, Maringá, vol.8, n¹, pp. 85-90, 1997.

ZORZI, Jaime. A inversão de letras na escrita: o fantasma do espelhamento. PróFono. 2001; 13(2):212-8. Disponível em: http://www.filologia.org.br/soletras/15sup/ As\%20invers\%C3\%B5es\%20de\%20letras $\% 20$ na\%20escrita- \%20o\%20'fantasma'\%20do\%20espelham ento.pdf. Recuperado em: agosto 25, 2011.

Recebido em: 30/09/2015 Aceito em: 25/11/2015 